\title{
Selective Hydrogenation of 5-Hydroxymethylfurfural via Zeolite Encapsulation to Avoid Further Hydrodehydroxylation
}

Qiang Chen, ${ }^{\text {ab }}$ Tianhao Li, ${ }^{\mathrm{b}}$ Yiming Zhou, ${ }^{\mathrm{b}}$ Yunfei Bi, ${ }^{\mathrm{c}}$ Shuai Guo, ${ }^{\mathrm{d}}$ Xuan Liu, ${ }^{\mathrm{b}}$ Haozhe

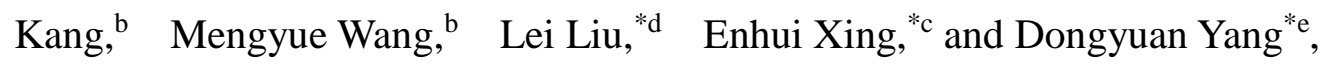

${ }^{a}$ School of Chemical Engineering and Technology, Sun Yat-sen University, Zhuhai campus, Zhuhai 519082, China

${ }^{b}$ School of Chemical Engineering and Technology, Xi'an Jiaotong University, Xi'an, Shaanxi 710049, China;

${ }^{c}$ Research Institute of Petroleum Processing, Sinopec, Beijing 100083, China

${ }^{d}$ Institute of Process Engineering, Chinese Academy of Sciences, Beijing 100190, China.

${ }^{e}$ Shaanxi yanchang Petroleum (Group) Corp. Ltd \& Dalian Institute of Chemical Physics Xi'an Clean Energy(chemical) Research Institute, Xi'an, Shaanxi 710065, China

*E-mail:

xingeh.ripp@sinopec.com (E. H. Xing);

liulei3039@gmail.com, liulei@ipe.ac.an (L. Liu);

yangdongyuan885@163.com (D. Y. Yang) 


\section{Table of Content}

Figure S1: XRD characterization of Pt@ Y catalyst.

Figure S2: HRTEM image of Pt@ Y catalyst.

Figure S3. HADDF-STEM image of the prepared Pt/Y catalyst and corresponding size distribution

Figure S4: HADDF-STEM images of Pt/Y catalyst before and after reaction

Table S1. Hydrogenation of different aldehyde groups over Pt@ Y catalyst. 


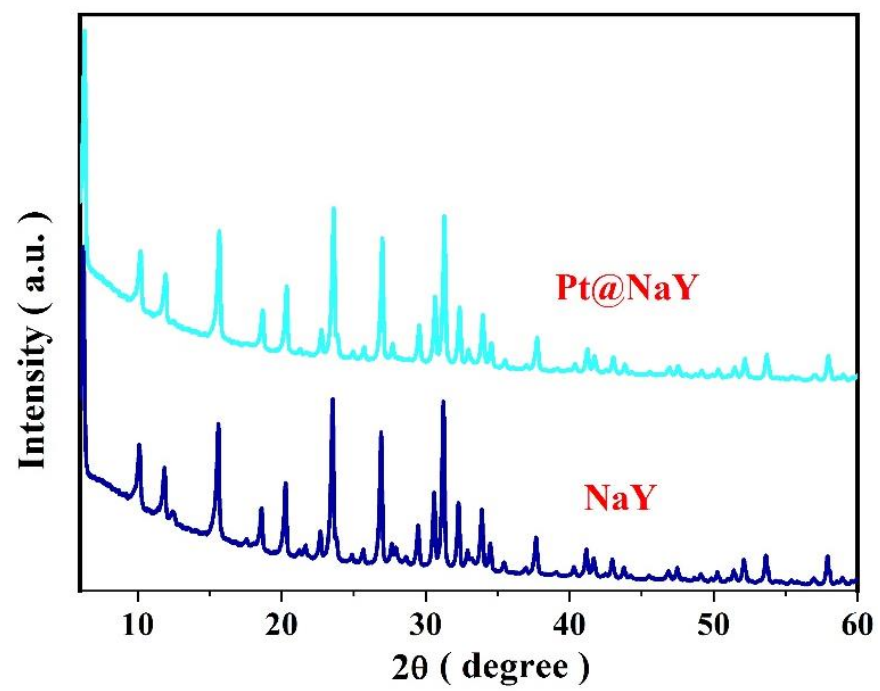

Figure S1: XRD characterization of Pt@Y catalyst. 


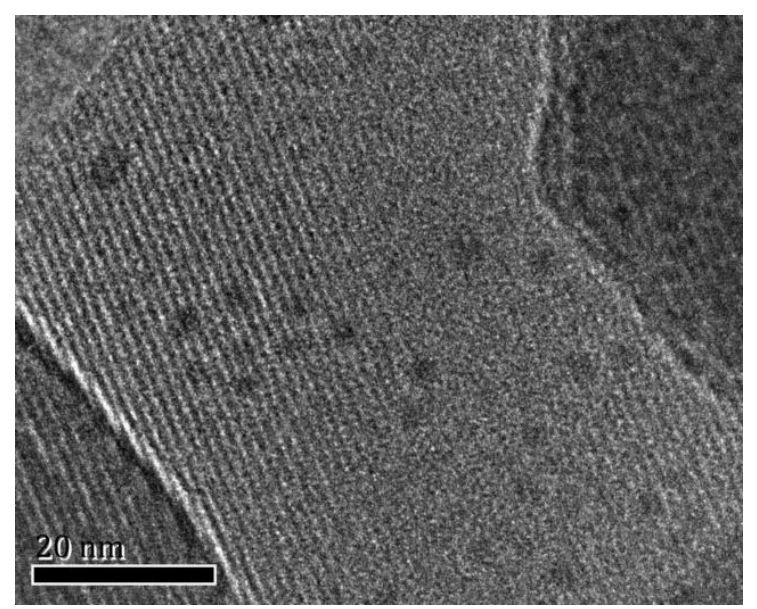

Figure S2: HRTEM image of Pt@ Y catalyst. 

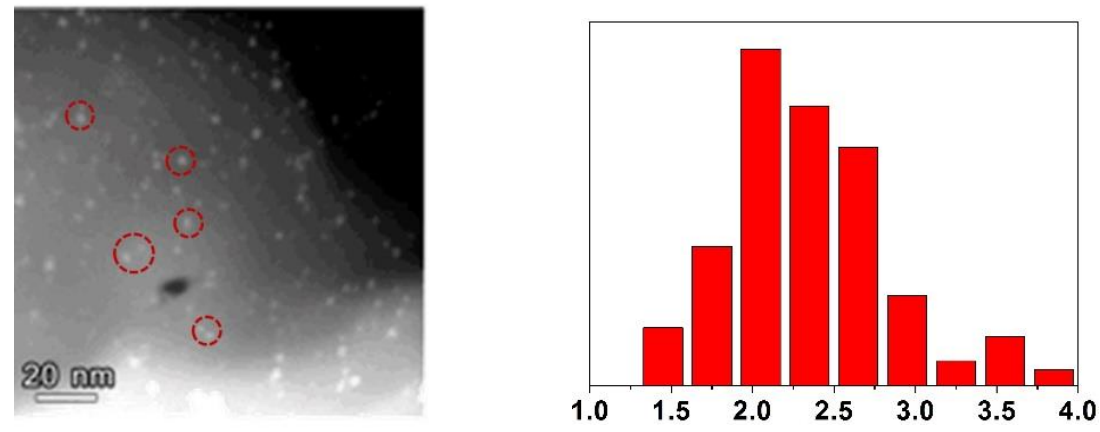

Figure S3. HADDF-STEM image of the prepared Pt/Y catalyst (left image), and the calculated average diameter size of PtNPs being $2.35 \mathrm{~nm}$ (right image). PtNPs were highlighted with red circles. 

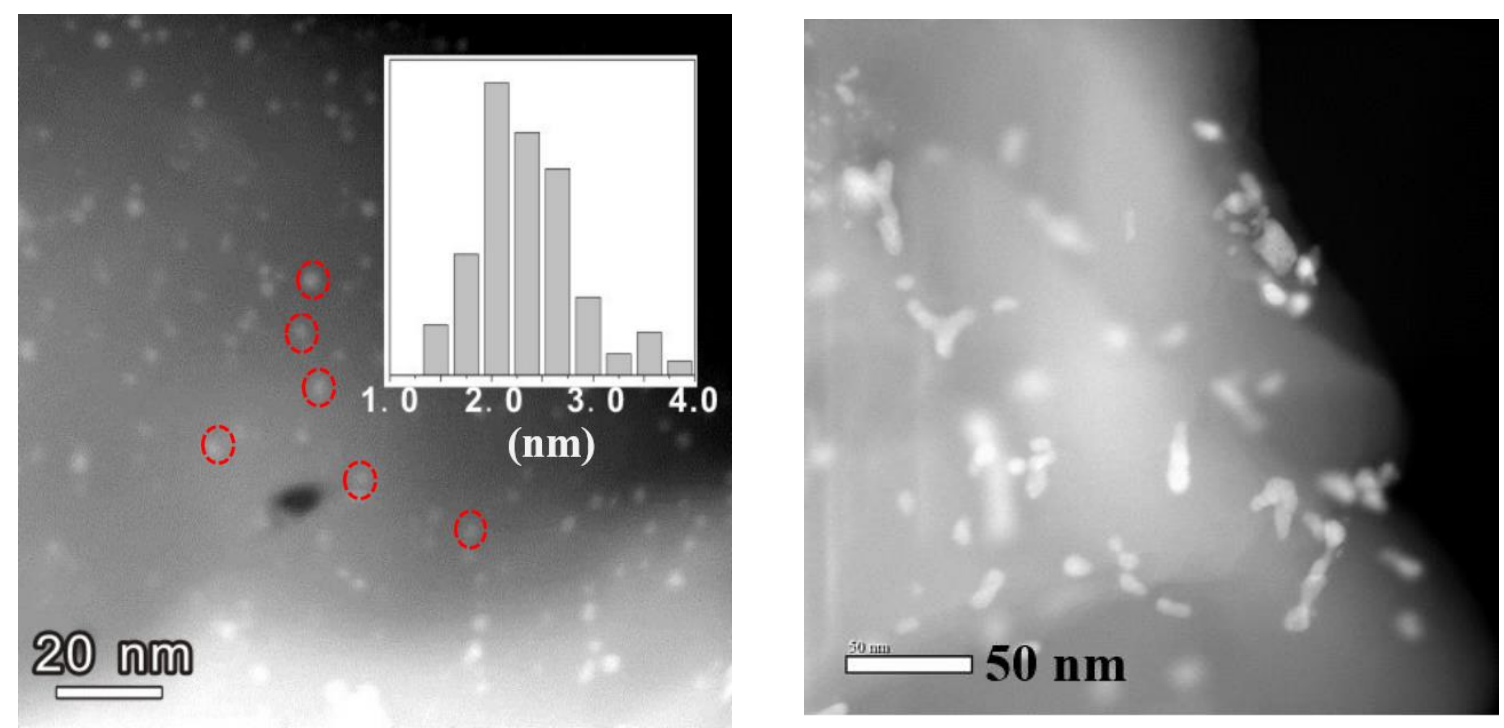

Figure S4: HADDF-STEM images of Pt/Y catalyst before (left) and after (right) reaction. PtNPs were highlighted with red circles. 
Table S1. Selective hydrogenation of different aldehyde groups to desired alcohol products over Pt@Y catalyst.

\begin{tabular}{cccc}
\hline Entry & substrate & Conv. $(\%)$ & Selectivity $(\%)$ \\
\hline 1 & $p$-ethyl-benzaldehyde & 100 & $>99$ \\
2 & heptanal & 100 & $>99$ \\
\hline
\end{tabular}

